\title{
Effective Hamiltonians of polymethineimine, polyazine and polyazoethene: A density matrix variation approach
}

\author{
GuanHua Chen ${ }^{\mathrm{a})}$ and ZhongMin Su \\ Department of Chemistry, University of Hong Kong, Pokfulam Road, Hong Kong \\ ZhenWen Shen and YiJing Yan ${ }^{\text {a) }}$ \\ Department of Chemistry, Hong Kong University of Science \& Technology, Kowloon, Hong Kong
}

(Received 5 March 1998; accepted 5 May 1998)

\begin{abstract}
A new variation method is proposed to determine the effective Hamiltonians for conjugated $\pi$-electron systems. This method is based on the minimization of the difference between the ground state reduced single electron density matrix calculated from the effective Hamiltonian and its $a b$ initio counterpart under a set of well-defined constraints. Applications are made to various oligomers of polymethineimine (PMI), polyazine (PAZ) and polyazoethene (PAE) at the HartreeFock level. Calculated are also the optical gaps of these oligomers. The effective Hamiltonians contain electron-electron Coulomb interactions and are suitable for the study of excited state dynamic processes such as nonlinear optical properties in $\pi$-conjugated systems. (C) 1998 American Institute of Physics. [S0021-9606(98)30630-3]
\end{abstract}

\section{INTRODUCTION}

There is a growing interest in evaluating the electronic dynamics of large and complex molecules. Ab initio calculations have been successful in determining ground and excited state properties of small and medium size molecular systems. However, calculating the properties, especially those that involve the excited state dynamics of large systems, requires a very large amount of computational resources. The burden of computation may be greatly reduced if only a fraction of electrons of a system is considered explicitly. The effects of other electrons may be included in an effective Hamiltonian. Pseudopotentials and effective core potentials have been developed to simulate the effects of core electrons. ${ }^{1-4}$ Pariser and Parr ${ }^{5}$ and Pople ${ }^{6}$ (PPP) devised an empirical procedure to determine the effective Hamiltonians for $\pi$ electrons in planar conjugated molecules. The parameters of the PPP Hamiltonians can be determined by theoretical fits to experimental data. Several parameterization schemes have been proposed. ${ }^{7,8}$ Semi-empirical methods such as CNDO, INDO, MNDO and AM1 employ empirical Hamiltonians in a minimal basis set of atomic orbitals. ${ }^{9-12}$ Like the PPP Hamiltonians, these Hamiltonians are parameterized to fit different sets of experiments.

Based on a formalism proposed by Brandow, ${ }^{13}$ Martin and Freed $^{14}$ have recently developed a rigorous projection method to investigate the foundation of PPP Hamiltonian. Demonstrations have been made in determining the effective Hamiltonians of several small conjugated molecules. ${ }^{14}$ In their method it requires the evaluation of some complex operators which are computationally intensive. ${ }^{14}$ Beratan and co-workers ${ }^{15,16}$ have adopted a divide-conquer strategy in which a large molecular system is treated as a collection of overlapping fragments. The effective Hamiltonian for the va-

${ }^{\text {a)} C o r r e s p o n d i n g ~ a u t h o r s . ~ E l e c t r o n i c ~ m a i l: ~ g h c @ y a n g t z e . k h u . h k ~}$ lence electrons of each individual fragment was constructed first, and that of the entire molecular system was then obtained by patching the contributions from all fragments.

Chen and Mukamel ${ }^{17}$ have shown that under certain circumstances there is a one-to-one correspondence between the one-electron part of the effective electronic Hamiltonian and its ground state reduced density matrix. As a Hamiltonian contains all dynamic information, the above theorem in fact also claims an effective correlation between the dynamic properties of excited states and the structural information of the ground electronic state. This is in the same spirit of the density functional theorem ${ }^{18}$ which states that the ground state electron density determines all properties of an electronic system. However, the new theorem that is based on a semi-empirical effective Hamiltonian description needs the ground state reduced density matrix as its input, whereas the density functional theorem requires a three-dimensional distribution of all electrons. Based on the new theorem, ${ }^{17}$ an approach at the Hartree-Fock level has been developed to construct the effective Hamiltonian from the ground state reduced single-electron density matrix. ${ }^{19}$ Application to polyacetylene oligomers $(-\mathrm{CH}=\mathrm{CH}-)_{x}$ has been made ${ }^{19}$ and the resulting effective Hamiltonians are consistent with those from the semi-empirical methods. $5,7,20$

In this work, we generalize the approach in Ref. 19 by proposing a new variation method to determine the effective Hamiltonian for $\pi$ electrons in conjugated polymer systems. This new method that will be developed in Sec. II is based on a constrained density matrix variation (CDMV) approach. The novelty of the method is to introduce a set of constraints that incorporate the structural information of polymers. As a result, the new method (CDMV) allows a more efficient approach to determine effective Hamiltonians and is applicable to more diversified conjugated polymer systems compared to the previous approach. ${ }^{19}$ Applications of the new method at the Hartree-Fock level are made in Sec. III to obtain the 
PMI<smiles></smiles><smiles></smiles>

PAE<smiles>C=CN=N/C=C/N=N[CH]N=N</smiles>

FIG. 1. Structures of PMI, PAZ and PAE. The numbers on PMI denote the $\pi$ atomic orbitals.

$\pi$-electron effective Hamiltonians for the oligomers of PAZ $(-\mathrm{CH}=\mathrm{N}-\mathrm{N}=\mathrm{CH}-)_{x}, \quad$ PMI $(-\mathrm{CH}=\mathrm{N}-)_{x}$ and PAE $(-\mathrm{CH}=\mathrm{CH}-\mathrm{N}=\mathrm{N}-)_{x}$. These polymers (Fig. 1) are structurally similar to polyacetylene (PA). The effective electronelectron Coulomb interactions in these conjugated $\pi$-electron systems are determined via a semi-empirical manner. The optical gaps of these polymer systems are evaluated to examine the validity of the new variation method and the reliability of the resulting effective Hamiltonians. In Sec. IV, we further compare our results to other calculations and conclude the present paper with a brief summary.

\section{A DENSITY MATRIX VARIATION APPROACH TO THE EFFECTIVE HAMILTONIAN}

\section{A. The general methodology}

The $\pi$ electrons in conjugated systems such as PMI, PAZ and PAE oligomers (cf. Fig. 1) may be described by the following PPP-like effective Hamiltonian:

$$
\begin{aligned}
& H=H_{e}+H_{e e}, \\
& H_{e}=\sum_{m n \sigma} t_{m n} a_{m \sigma}^{+} a_{n \sigma}, \\
& H_{e e}=\sum_{m n \sigma \sigma^{\prime}} V_{m n} a_{m \sigma}^{+} a_{m \sigma} a_{n \sigma^{\prime}}^{+} a_{n \sigma^{\prime}} .
\end{aligned}
$$

Here, $a_{m \sigma}^{+}\left(a_{n \sigma}\right)$ is an electron creation (annihilation) operator at a local orbital $m(n)$ with spin $\sigma . t_{m n}$ denotes the one-electron on-site $(m=n)$ or resonance $(m \neq n)$ integral. $V_{m n}$ is the effective two-electron Coulomb interaction between two electrons residing separately at $m$ and $n$. Therefore, $H_{e}$ represents the one-electron part of the Hamiltonian which describes the dynamics of each individual $\pi$ electrons in the absence of other $\pi$ electrons. $H_{e e}$ is the two-electron contribution which represents the effective Coulomb interactions among electrons. A zero differential overlap (ZDO) approximation $^{21}$ is adopted here. The nuclear charge for every site is +1 . Since the systems are spin symmetric, we may omit the spin index $\sigma$ or $\sigma^{\prime}$. We have thus converted the quest of an effective Hamiltonian to the problem of finding an optimal set of parameters $\left\{t_{m n}, V_{m n}\right\}$ in Eq. (1). In the following, we shall present a constrained density matrix variation (CDMV) approach to determine the effective Hamiltonian.

Let us denote $\rho$ as the ground state density matrix from the effective Hamiltonian $H$ [Eq. (1)], and $\bar{\rho}$ as that from an $a b$ initio calculation. The accuracy of $\rho$ together with the effective Hamiltonian may be characterized by the following CDMV functional:

$$
S=\sum_{m n} W_{m n}\left(\rho_{m n}-\bar{\rho}_{m n}\right)^{2}+\mathcal{F} \geqslant 0 .
$$

Here, $W_{m n}$ is a positive weighting (or penalty) coefficient for the deviation of the semi-empirical density matrix element $\rho_{m n}$ from its ab initio counterpart $\bar{\rho}_{m n} . \mathcal{F}$, which will be presented later [cf. Eq. (6)], consists of all additional constraints implied to the form of the effective Hamiltonian.

We adopt the Hückel's ansatz for the one-electron contribution $H_{e}$ [Eq. (1b)]. That is to set all non-neighboring resonance integrals to zero:

$$
t_{m n}=0 ; \text { if }|m-n|>1 .
$$

Simplifying the notation, we shall hereafter denote the neighboring resonance integral as

$$
t_{m}^{\prime} \equiv t_{m, m+1} \text {. }
$$

We shall further introduce $t_{m}^{*}$ as the effective atomic orbital energy at the local orbital $m$ in the presence of electronnuclear Coulomb interaction. It is given by ${ }^{19}$

$$
t_{m}^{*} \equiv t_{m m}+\sum_{n}\left(V_{m n}-V_{1 n}\right)
$$

As only the relative energies are of physical interest, we can choose the energy zero as $t_{11}=0$, which also results in $t_{1}^{*}$ $=0$.

We are now in a position to discuss the constraint functional $\mathcal{F}$ in Eq. (2). We propose that the form of the effective Hamiltonian which characterizes the polymer configurations of interest should be retained as much as possible during the functional minimization process. We can thus present $\mathcal{F}$ in the following form: ${ }^{19}$

$$
\mathcal{F}=W_{1} \sum_{m}\left(t_{m}^{*}-t_{m+s}^{*}\right)^{2}+W_{2} \sum_{m}\left(t_{m}^{\prime}-t_{m+s}^{\prime}\right)^{2} .
$$

Here, $s$ is the length of the repeating unit in the polymer. For examples, $s=4$ for PAZ $(-\mathrm{CH}=\mathrm{N}-\mathrm{N}=\mathrm{CH}-)_{x}$ and PAE $(-\mathrm{CH}=\mathrm{CH}-\mathrm{N}=\mathrm{N}-)_{x}$, and $s=2$ for PMI $(-\mathrm{CH}=\mathrm{N}-)_{x} . W_{1}$ and $W_{2}$ are two positive penalty constants. The first and second 
terms in Eq. (6) penalize the mismatches of the effective orbital energies and resonance integrals, respectively, in the repeating units of the polymer.

In principle, $S=0$ in Eq. (2) can only be achieved when the effective Hamiltonian produces the exact ground density matrix $\bar{\rho}$ and at the same time the constraints are also satisfied. For a semi-empirical Hamiltonian as Eq. (1), this ideal case is practically impossible. We shall thus optimize the effective Hamiltonian $H$ [Eq. (1)] or more precisely the set of parameters $\left\{t_{m n}, V_{m n}\right\}$ that characterizes $H$, so that the resulting reduced ground state density matrix $\rho$ optimally overlaps with the $a b$ initio reduced density matrix $\bar{\rho}$. In this case, the CDMV functional $S$ [Eq. (2)] is minimal.

\section{B. Monte Carlo implementation in the Hartree-Fock scheme}

We shall now present in detail a Hartree-Fock scheme of implementing the above CDMV approach. The $a b$ initio ground state density matrix $\bar{\rho}$ at the Hartree-Fock level is evaluated first and saved for the later reference in constructing the CDMV functional $S$ [Eq. (2)]. The Hartree-Fock equation for an effective Hamiltonian $H$ is

$$
\sum_{n} h_{m n} c_{n i}=\epsilon_{i} c_{m i}
$$

Here, $c_{m i}$ is the coefficient of local atomic orbital $m$ in the $i$-th molecular orbital with energy $\epsilon_{i}$. The Fock matrix element $h_{m n}$ in Eq. (7) may be expressed as ${ }^{22}$

$$
h_{m n}=t_{m n}-V_{m n} \rho_{m n}+2 \delta_{m n} \sum_{l} V_{m l} \rho_{l l} .
$$

For a system of $M \pi$ electrons, the ground state reduced single electron density matrix $\rho$ may then be evaluated as

$$
\rho_{m n}=\sum_{i}^{M / 2} c_{n i}^{*} c_{m i} .
$$

Equations (7)-(9) summarize the Hartree-Fock approach to calculate the ground state electron density matrix $\rho$ for a given effective Hamiltonian.

In order to find the optimal effective Hamiltonian $H$ that minimizes the CDMV functional $S$ [Eq. (2)], we employ an annealing Monte Carlo procedure as described follows. Beginning with an initial guess $H^{(0)}$ for the effective Hamiltonian $H$, we solve for its Hartree-Fock ground state density matrix $\rho^{(0)}$ via Eqs. (7)-(9), and then evaluate the CDMV functional $S^{(0)}$ using Eq. (2) for the chosen values of $W_{m n}$, $W_{1}$ and $W_{2}$. We shall now (i) vary randomly the current parameters in $H^{(0)}$ to generate a trial $H^{(1)}$; (ii) evaluate $\rho^{(1)}$ via Eqs. (7)-(8) and $S^{(1)}$ [Eq. (2)] for the trial $H^{(1)}$; (iii) and decide whether to retain $H^{(0)}$ or to accept $H^{(1)}$ for the next step of iteration according to their survival probabilities, which are assumed to be proportional to their corresponding values of $\exp \left[-S^{(0)} / T\right]$ and $\exp \left[-S^{(1)} / T\right]$, respectively. Here, $T>0$ is the simulation temperature. The above procedure of (i)-(iii) shall be repeated many times to generate (statistically) better and better approximations, and eventually leads to the true effective Hamiltonian $H$. During the process of $S$ minimization, $T$ is varied from high to low values, and eventually approaches zero. In all calculations to be described in the next section, we set $W_{m n}=1.0$ and $W_{1}=W_{2}=0.1$. Six different values of the temperature $T$ are used: $T$ $=1.0,10^{-1}, 10^{-2}, 10^{-3}, 10^{-4}$ and $10^{-5}$. At each temperature the above process is repeated a number $(N)$ of times. $N$ $=200,300,1500,500,100$ and 100 for these six temperatures, respectively.

It is obviously that the efficiency of above mentioned CDMV approach depends greatly on the number of the Hamiltonian parameters involving in the minimization procedure. We have used the Hückel's ansatz [Eq. (3)] which greatly reduces the number of one-electron parameters $\left\{t_{m n}\right\}$. In the following section, we shall focus on PAZ $(-\mathrm{CH}=\mathrm{N}-\mathrm{N}=\mathrm{CH}-)_{x}, \quad$ PMI $(-\mathrm{CH}=\mathrm{N}-)_{x}$ and PAE $(-\mathrm{CH}=\mathrm{CH}-\mathrm{N}=\mathrm{N}-)_{x}$ systems, in which the Coulomb interactions $V_{m n}$ will be determined in a semi-empirical manner [cf. Eq. (10)]. As a result, the parameters $\left\{V_{m n}\right\}$ will no longer be considered as the minimization variables. The final survival variables are the diagonal one-electron integrals and the nearest-neighboring resonances [cf. Eq. (4)], $\left\{t_{m m}, t_{m-1}^{\prime} ; m\right.$ $=2, \ldots, M\}$. Here $M$ is the number of the $\pi$ orbitals in consideration.

\section{APPLICATIONS TO PMI, PAZ AND PAE OLIGOMERS}

\section{A. Semi-empirical approach to electron-electron interaction}

Let us start with a semi-empirical method to determine the effective Coulomb interaction $V_{m n}$ in PMI $(-\mathrm{CH}=\mathrm{N}-)_{x}$, $\mathrm{PAZ}(-\mathrm{CH}=\mathrm{N}-\mathrm{N}=\mathrm{CH}-)_{x}$ and $\mathrm{PAE}(-\mathrm{CH}=\mathrm{CH}-\mathrm{N}=\mathrm{N}-)_{x}$ systems. The effective Coulomb interaction is usually unknown. However, its functional form may be derived from manybody theory or determined empirically. In this work, we adopt the Ohno formula to describe the effective Coulomb interaction: ${ }^{23}$

$$
V_{m n}=\frac{U_{\mathrm{AB}}}{\sqrt{1+\left(r_{m n} / a_{0}\right)^{2}}} .
$$

Here, $r_{m n}$ is the distance between the $\pi$ orbitals $m$ and $n$. A and $\mathrm{B}$ denote the atoms that in this work may be carbon $\mathrm{C}$ or nitrogen $\mathrm{N}$ where the orbitals $m$ and $n$ reside, respectively. $U_{\mathrm{AB}}$ determines the overall amplitude of interaction between two electrons located at atoms A and B separately. Its value may be considered as the effective on-site repulsion if the two electrons are on the same atom [cf. Eq. (10)]. It has been demonstrated that the Ohno formula describes well the effective interaction among $\pi$ electrons in conjugated polymers. ${ }^{7,19}$ In Ref. 19, the Ohno formula was applied to the $\pi$ electrons in polyacetylene, and the value of $U_{\mathrm{CC}}$ was determined by fitting to the experimental optical gap of $(-\mathrm{CH}=\mathrm{CH}-)_{7}$ oligomer. It has also been illustrated that the effective Hamiltonian is not sensitive to the value of $a_{0}$ as long as it is of the same magnitude as the bond length. ${ }^{19} \mathrm{We}$ set $a_{0}=1.0 \AA$ for all three types of polymers in study. $r_{m n}$ in Eq. (10) will be determined via the $a b$ initio calculation for each individual oligomer. The Coulomb repulsion amplitude $U_{\mathrm{CN}}$ or $U_{\mathrm{NC}}$ will be approximated as 


$$
U_{\mathrm{CN}}=U_{\mathrm{NC}} \approx \sqrt{U_{\mathrm{CC}} U_{\mathrm{NN}}}
$$

In Ref. 19, the repulsion amplitude $U_{\mathrm{CC}}$ for two $\pi$ electrons in carbon atoms was determined to be $6.62 \mathrm{eV}$. We shall evaluate $U_{\mathrm{NN}}$ using the CDMV approach developed in the last section.

\section{B. Determination of $\boldsymbol{U}_{\mathrm{NN}}$}

Let us consider a theoretical oligomer $(-\mathrm{N}=\mathrm{N}-)_{7}$. The first step of the CDMV approach is to construct its ab initio ground density matrix $\bar{\rho}$. We use Gaussian 94 with the minimal basis set STO-3G in our calculation. From the resulting Hartree-Fock ground state wave function, we derive the corresponding reduced density matrix $\bar{\rho}$ of the oligomer in the Natural Atomic Orbitals (NAOs) basis set. ${ }^{24}$ The NAOs are the orthonormal atomic orbitals of maximal occupancy for a given wave function. ${ }^{25}$ They diagonalize the atomic reduced single-electron density matrix, and thus provide a compact representation of electronic properties. During the process of obtaining the $a b$ initio ground density matrix, the geometry of $(-\mathrm{N}=\mathrm{N}-)_{7}$ is optimized and thus the value of $r_{m n}$ in Eq. (10) is obtained for each pair of $\pi$ orbitals. The ground state is found to be a trans structure. The resulting bond lengths of the double and single bonds are $1.27 \AA$ and $1.49 \AA$, respectively. The angles between two adjacent bonds is $105.3^{\circ}$.

We are now in the position to apply the CDMV approach described in Sec. II B to determine the effective Hamiltonian of the theoretical molecule $(-\mathrm{N}=\mathrm{N}-)_{7}$ which contains $14 \pi$ electrons, each from a nitrogen atom. The effective Hamiltonian in this case refers to the two-electron parameter $U_{\mathrm{NN}}$ together with the complete set of oneelectron parameters $\left\{t_{m m}, t_{m-1}^{\prime} ; m=2, \ldots, 14\right\}$ in the Hückel's ansatz [Eq. (3)]. By employing the Ohno formula [Eq. (10)], we can determine the effective Hamiltonians of $(-\mathrm{N}=\mathrm{N}-)_{7}$ up to an overall undetermined scaling factor. In order to determine the value of this scaling factor, one physical quantity such as the optical gap $\Delta_{\mathrm{N}}$ (i.e., absorption peak) is required. ${ }^{19}$ As $(-\mathrm{N}=\mathrm{N}-)_{7}$ is not available experimentally, we estimated $\Delta_{\mathrm{N}}$ via the following relation:

$$
\Delta_{\mathrm{N}}=\frac{\delta E_{\mathrm{N}}}{\delta E_{\mathrm{C}}} \Delta_{\mathrm{C}}
$$

Here, $\delta E_{\mathrm{N}}=11.16 \mathrm{eV}$ is the energy difference between the LUMO and HOMO of $(-\mathrm{N}=\mathrm{N}-)_{7}$ molecule evaluated via the ab initio calculation, while $\delta E_{\mathrm{C}}=10.26 \mathrm{eV}$ is that for (-CH $=\mathrm{CH}-)$ obtained previously. ${ }^{19}$ The experimental value of the optical gap $\Delta_{\mathrm{C}}$ is $3.18 \mathrm{eV} .{ }^{26}$ We have therefore $\Delta_{\mathrm{N}}$ $=3.46 \mathrm{eV}$, and can thus determine unambiguously the effective Hamiltonian of $(-\mathrm{N}=\mathrm{N}-)_{7}$ such that it reproduces the optical gas of $\Delta_{\mathrm{N}}=3.46 \mathrm{eV}$. The average values of the resulting resonance integrals through the double and single bonds are $t_{d}=-2.42 \mathrm{eV}$ and $t_{s}=-1.30 \mathrm{eV}$, respectively. The corresponding value of $U_{\mathrm{NN}}$ is $4.72 \mathrm{eV}$. The coupled electronic oscillator (CEO) method ${ }^{22,27}$ is employed in the evaluation of the optical gap.
TABLE I. Structure parameters of PMI, PAZ and PAE. ${ }^{\mathrm{a}}$

\begin{tabular}{lccc}
\hline \hline & PMI & PAZ & PAE \\
\hline$R_{\mathrm{C}=\mathrm{N}}$ & 1.264 & 1.288 & - \\
$R_{\mathrm{C}-\mathrm{N}}$ & 1.390 & - & 1.464 \\
$R_{\mathrm{C}=\mathrm{C}}$ & - & - & 1.322 \\
$R_{\mathrm{C}-\mathrm{C}}$ & - & 1.473 & - \\
$R_{\mathrm{N}=\mathrm{N}}$ & - & - & 1.281 \\
$R_{\mathrm{N}-\mathrm{N}}$ & - & 1.473 & - \\
$\alpha$ & 120.2 & 113.7 & 119.5 \\
\hline \hline
\end{tabular}

${ }^{\mathrm{a} B o n d}$ lengths $R_{\mathrm{C}=\mathrm{N}}, R_{\mathrm{C}-\mathrm{N}}, R_{\mathrm{C}=\mathrm{C}}, R_{\mathrm{C}-\mathrm{C}}, R_{\mathrm{N}=\mathrm{N}}$ and $R_{\mathrm{N}-\mathrm{N}}$ are in units of $\AA$; bond angle $\alpha$ between adjacent double and single bonds in degrees.

\section{Effective Hamiltonians for various oligomers}

Having obtained the values of $U_{\mathrm{NN}}=4.72 \mathrm{eV}, U_{\mathrm{CC}}$ $=6.62 \mathrm{eV}$ and $U_{\mathrm{CN}} \approx \sqrt{U_{\mathrm{CC}} U_{\mathrm{NN}}}=5.59 \mathrm{eV}$, we are now ready to evaluate the effective Hamiltonians of PMI, PAZ and PAE oligomers via the procedure described in Sec. II B. We first determine the structures of PMI, PAZ and PAE oligomers via the $a b$ initio Hartree-Fock geometry optimization calculation. The 6-31G basis set is employed. For PMI oligomers, we choose $\mathrm{H}_{2} \mathrm{C}=\mathrm{N}-(\mathrm{HC}=\mathrm{N})_{6}-\mathrm{N}=\mathrm{CH}_{2}$ for optimization. The lengths for all the double $(\mathrm{C}=\mathrm{N})$ bonds are set equal, and those for all the single $(\mathrm{C}-\mathrm{N})$ bonds are as well. The bond angles for all $\mathrm{CNC}$, (NCN) or $\mathrm{HCN}$ are kept the same. The resulting structure is similar to that used by Champagne and co-workers. ${ }^{28}$ Similarly, we obtain the optimized structures of $\mathrm{H}_{2} \mathrm{C}=\mathrm{N}-\mathrm{N}=\mathrm{CH}-(\mathrm{HC}=\mathrm{N}-\mathrm{N}=\mathrm{CH})_{2}-\mathrm{CH}=\mathrm{N}-\mathrm{N}=\mathrm{CH}_{2}$ and $\mathrm{H}_{2} \mathrm{C}=\mathrm{CH}-\mathrm{N}=\mathrm{N}-(\mathrm{HC}=\mathrm{CH}-\mathrm{N}=\mathrm{N})_{2}-\mathrm{CH}=\mathrm{CH}-\mathrm{N}=\mathrm{NH}_{2}$. The resulting structure parameters for the above three polymer molecules are listed in Table I. These parameters will be employed, respectively, for all PMI, PAZ and PAE oligomers in later calculations. The $a b$ initio Hartree-Fock ground state density matrix $\bar{\rho}$ for each oligomer of the transPMI, PAZ and PAE with 2 to 20 double bonds is calculated by using the same procedure described in the last subsection for $(-\mathrm{N}=\mathrm{N}-)_{7}$. Note that we use the STO-3G basis set for evaluating the $a b$ initio density matrices, instead of the 6$31 \mathrm{G}$ that was used in the geometry optimization. The effective Hamiltonians for all these oligomers are then determined accordingly via the CDMV approach described in Sec. II B. Listed in Table II are the three sets of initial values of $t_{i j}$ used for all PMI, PAZ and PAE oligomers. For the same type oligomers, e.g. $(-\mathrm{CH}=\mathrm{N}-)_{x}$, only one set of initial $t_{i j}$ is employed for all with different repeating units.

TABLE II. Initial guess of $t_{i j}$ for PMI, PAZ and PAE oligomers (in eV). ${ }^{\mathrm{a}}$

\begin{tabular}{cccccccc}
\hline \hline & $t^{*}(\mathrm{~N})$ & $t(\mathrm{C}=\mathrm{N})$ & $t(\mathrm{C}-\mathrm{N})$ & $t(\mathrm{C}=\mathrm{C})$ & $t(\mathrm{C}-\mathrm{C})$ & $t(\mathrm{~N}=\mathrm{N})$ & $t(\mathrm{~N}-\mathrm{N})$ \\
\hline PMI & -1.0 & -3.1 & -2.1 & - & - & - & - \\
PAZ & -1.6 & -3.3 & - & - & -1.9 & - & -1.9 \\
PAE & -1.3 & - & -2.2 & -3.1 & - & -3.5 & - \\
\hline \hline
\end{tabular}

${ }^{\mathrm{a}} t *(\mathrm{~N})$ is the effective atomic energy of the local orbital on $\mathrm{N}, t(\mathrm{C}=\mathrm{N})$, $t(\mathrm{C}-\mathrm{N}), t(\mathrm{C}=\mathrm{C}), t(\mathrm{C}-\mathrm{C}), t(\mathrm{~N}=\mathrm{N})$, and $t(\mathrm{~N}-\mathrm{N})$ are the resonance integrals for $\mathrm{C}=\mathrm{N}, \mathrm{C}-\mathrm{N}, \mathrm{C}=\mathrm{C}, \mathrm{C}-\mathrm{C}, \mathrm{N}=\mathrm{N}$ and $\mathrm{N}-\mathrm{N}$, respectively, and the effective atomic energy $t^{*}(\mathrm{C})$ was set to zero. 
TABLE III. Effective Hamiltonians of PMI oligomers $(-\mathrm{CH}=\mathrm{N}-)_{x} \cdot{ }^{\mathrm{a}}$

\begin{tabular}{rcccc}
\hline \hline$x$ & $t_{d} / \mathrm{eV}$ & $t_{s} / \mathrm{eV}$ & $t^{*}(\mathrm{C})-t^{*}(\mathrm{~N}) / \mathrm{eV}$ & $\Delta / \mathrm{eV}$ \\
\hline 2 & -3.13 & -2.20 & 1.03 & 5.57 \\
4 & -3.11 & -2.19 & 1.13 & 4.23 \\
8 & -3.11 & -2.19 & 1.17 & 3.49 \\
12 & -3.11 & -2.19 & 1.19 & 3.27 \\
16 & -3.11 & -2.19 & 1.19 & 3.19 \\
20 & -3.11 & -2.19 & 1.20 & 3.15 \\
\hline \hline
\end{tabular}

${ }^{\mathrm{a}} t_{d}$ and $t_{s}$ are the average resonance integrals for $\mathrm{C}=\mathrm{N}$ and $\mathrm{N}-\mathrm{C}$, respectively. $t^{*}(\mathrm{C})$ and $t^{*}(\mathrm{~N})$ are the average effective atomic orbital energies for $\pi$ orbitals of $\mathrm{C}$ and $\mathrm{N}$ atoms, respectively. $\Delta$ is the optical gap.

In Tables III, IV and $\mathrm{V}$, we list the resulting parameters in the effective Hamiltonians of PMI, PAZ and PAE oligomers, respectively. These data are reported in terms of the average resonance integrals $t_{d}$ and $t_{s}$ for the double and single bonds, respectively, and the average effective atomic orbital energy difference $t^{*}(\mathrm{C})-t^{*}(\mathrm{~N})$ between the $\pi$ orbitals on $\mathrm{C}$ and $\mathrm{N}$ atoms. In Table $\mathrm{IV}, t_{s}(\mathrm{C}-\mathrm{C})$ and $t_{s}(\mathrm{~N}-\mathrm{N})$ are both listed for the two types of single bonds in PAZ; whereas in Table $\mathrm{V}, t_{d}(\mathrm{C}=\mathrm{C})$ and $t_{d}(\mathrm{~N}=\mathrm{N})$ are for the two types of double bonds in PAE. The mean difference $\left|t_{d}-t_{s}\right|$ between the double and single bonds are $0.92 \mathrm{eV}$ in $(-\mathrm{CH}=\mathrm{N}-)_{20}$, $1.28 \mathrm{eV}$ in $(-\mathrm{CH}=\mathrm{N}-\mathrm{N}=\mathrm{CH}-)_{10}$ and $1.21 \mathrm{eV}$ in $(-\mathrm{CH}=\mathrm{CH}-\mathrm{N}=\mathrm{N}-)_{10}$, respectively, as indicated in the last rows of Tables III, IV and V. Note the values of $t^{*}(\mathrm{C})$ $-t^{*}(\mathrm{~N})$ of the PMI oligomers (cf. Table III) range from 1.03 to $1.20 \mathrm{eV}$, which are consistent with the result of $1.06 \mathrm{eV}$ obtained recently by Jacquemin and co-workers. ${ }^{29}$ Comparing the data in Tables III, IV and V, we observe that PAZ oligomers have the largest average effective atomic orbital energy difference $(1.6 \mathrm{eV})$. Included in these tables are also the optical gap $\Delta$ calculated via the CEO method ${ }^{22,27}$ for every individual oligomer. At any given number of double bonds, the PAZ oligomer possesses the largest optical gap. This may be explainable by the fact that the PAZ oligomer have larger resonance integral difference $\left|t_{d}-t_{s}\right|$ compared with those of PAZ and PAE counterparts. For $(-\mathrm{CH}=\mathrm{N}-\mathrm{N}=\mathrm{CH}-)_{20}$, its optical gap is $3.89 \mathrm{eV}$, and the corresponding wavelength is $3190 \AA$ which is outside the visible wavelength range. Thus, PAZ is apparently transparent.

TABLE IV. Effective Hamiltonians of PAZ Oligomers $(-\mathrm{CH}=\mathrm{N}-\mathrm{N}=\mathrm{CH}-)_{x} \cdot{ }^{\mathrm{a}}$

\begin{tabular}{rccccc}
\hline \hline$x$ & $t_{d} / \mathrm{eV}$ & $t_{s}(\mathrm{~N}-\mathrm{N}) / \mathrm{eV}$ & $t_{s}(\mathrm{C}-\mathrm{C}) / \mathrm{eV}$ & $t^{*}(\mathrm{C})-t^{*}(\mathrm{~N}) / \mathrm{eV}$ & $\Delta / \mathrm{eV}$ \\
\hline 1 & -3.35 & -1.89 & & 1.58 & 6.35 \\
2 & -3.45 & -1.94 & -2.17 & 1.62 & 5.04 \\
4 & -3.21 & -1.82 & -2.03 & 1.61 & 4.12 \\
6 & -3.21 & -1.82 & -2.03 & 1.62 & 3.96 \\
8 & -3.20 & -1.82 & -2.03 & 1.62 & 3.91 \\
10 & -3.21 & -1.83 & -2.04 & 1.62 & 3.89 \\
\hline \hline
\end{tabular}

${ }^{\mathrm{a}} t_{d}, t_{s}(\mathrm{~N}-\mathrm{N})$ and $t_{s}(\mathrm{C}-\mathrm{C})$ are the average resonance integrals for $\mathrm{C}=\mathrm{N}$, $\mathrm{N}-\mathrm{N}$ and $\mathrm{C}-\mathrm{C}$, respectively. $t^{*}(\mathrm{C})$ and $t^{*}(\mathrm{~N})$ are the average effective atomic orbital energies for the $\pi$ orbitals of $\mathrm{C}$ and $\mathrm{N}$ atoms, respectively. $\Delta$ is the optical gap.
TABLE V. Effective Hamiltonian of the PAE oligomer $(-\mathrm{CH}=\mathrm{CH}-\mathrm{N}=\mathrm{N}-)_{x} \cdot{ }^{a}$

\begin{tabular}{rccccc}
\hline \hline$x$ & $t_{d}(\mathrm{C}=\mathrm{C}) / \mathrm{eV}$ & $t_{d}(\mathrm{~N}=\mathrm{N}) / \mathrm{eV}$ & $t_{s} / \mathrm{eV}$ & $t^{*}(\mathrm{C})-t^{*}(\mathrm{~N}) / \mathrm{eV}$ & $\Delta / \mathrm{eV}$ \\
\hline 1 & -3.18 & -3.47 & -2.14 & 1.38 & 5.95 \\
2 & -3.05 & -3.69 & -2.13 & 1.38 & 4.61 \\
4 & -3.01 & -3.69 & -2.11 & 1.39 & 3.85 \\
6 & -3.00 & -3.65 & -2.11 & 1.38 & 3.74 \\
8 & -2.99 & -3.64 & -2.11 & 1.38 & 3.69 \\
10 & -3.00 & -3.63 & -2.11 & 1.39 & 3.67 \\
\hline \hline
\end{tabular}

${ }^{\mathrm{a}} t_{d}(\mathrm{C}=\mathrm{C}), t_{d}(\mathrm{~N}=\mathrm{N})$ and $t_{s}$ are the average resonance integrals for $\mathrm{C}=\mathrm{C}$, $\mathrm{N}=\mathrm{N}$ and $\mathrm{C}-\mathrm{N}$ (or $\mathrm{N}-\mathrm{C}$ ), respectively; $t^{*}(\mathrm{C})$ and $t^{*}(\mathrm{~N})$ are the average effective atomic orbital energies for the $\pi$ orbitals of $\mathrm{C}$ and $\mathrm{N}$ atoms, respectively. $\Delta$ is the optical gap.

\section{DISCUSSION AND CONCLUSION}

The optical gaps of PMI, PAZ and PAE were previously obtained via the $a b$ initio crystal orbital calculation. ${ }^{30}$ For PMI, it was also evaluated in the $\mathrm{MNDO}^{31}$ and valence effective Hamiltonian $(\mathrm{VEH})^{32}$ schemes. In Table VI, we compare our resulting optical gaps of PMI, PAZ and PAE with those obtained earlier via other approaches. Our results are chosen from the oligomers that contain 20 double bonds. As can be seen, the optical gap of each polymer molecule obtained from the present work is much smaller than that of the $a b$ initio or VEH calculation, but closer to the MNDO's result. Noted that the same $a b$ initio crystal orbital calculation $^{30}$ resulted in the optical gap of $6.47 \mathrm{eV}$ in polyacetylene (PA). In contrast, a calculation based on the same CEO method ${ }^{27,22}$ as this work led to the experimental optical gap of about $2 \mathrm{eV}$ in PA. Using the effective Hamiltonians obtained in this work to study the nonlinear optical properties of the PMI, PAZ and PAE oligomers has been carried out and the results will be published elsewhere. ${ }^{33}$

Jacquemin and co-workers ${ }^{29}$ employed a PPP-like effective Hamiltonian in their calculation of static hyperpolarizabilities of PMI oligomers. The energies of the atomic orbitals of $\mathrm{C}$ and $\mathrm{N}$ were used to approximate the effective atomic orbital energies of $\pi$ orbitals at $\mathrm{C}$ and $\mathrm{N}$ sites, respectively. This approximation led to $t^{*}(\mathrm{C})-t^{*}(\mathrm{~N})=1.06 \mathrm{eV}$, regardless the size of $(-\mathrm{CH}=\mathrm{N}-)_{x}$ oligomer. In the present work, the values of $t^{*}(\mathrm{C})-t^{*}(\mathrm{~N})$ increase from 1.03 to $1.20 \mathrm{eV}$ as the oligomer size increases (cf. Table III). For the resonance integrals, two approximations were used in Ref. 29. One was proposed by Pariser and Parr, ${ }^{5}$

$$
t_{i j}=-6442 e^{-5.6864 r_{i j}} \mathrm{eV},
$$

TABLE VI. A comparison of the optical gaps of PMI, PAZ and PAE (in units of $\mathrm{eV}$ ).

\begin{tabular}{ccccc}
\hline \hline & Our result & Ab $_{\text {initio }}^{\mathrm{a}}$ & $\mathrm{MNDO}^{\mathrm{b}}$ & $\mathrm{VEH}^{\mathrm{c}}$ \\
\hline PMI & 3.15 & 8.48 & 3.46 & 5.4 \\
PAZ & 3.89 & 9.56 & - & - \\
PAE & 3.67 & 9.62 & - & - \\
\hline \hline
\end{tabular}

${ }^{\text {aFrom Ref. } 30 .}$

${ }^{\mathrm{b}}$ From Ref. 31.

${ }^{c}$ From Ref. 32. 
TABLE VII. Five structural variations of the PMI oligomer $(-\mathrm{CH}=\mathrm{N}-)_{10} \cdot{ }^{\mathrm{a}}$

\begin{tabular}{lccccc}
\hline \hline & $\mathbf{a}$ & $\mathbf{b}$ & $\mathbf{c}$ & $\mathbf{d}$ & $\mathbf{e}$ \\
\hline$R_{\mathrm{C}=\mathrm{N}}$ & 1.277 & 1.264 & 1.252 & 1.232 & 1.217 \\
$R_{\mathrm{C}-\mathrm{N}}$ & 1.377 & 1.390 & 1.402 & 1.422 & 1.437 \\
$\Delta R$ & 0.100 & 0.126 & 0.150 & 0.190 & 0.220 \\
\hline \hline
\end{tabular}

${ }^{a}$ All data are given in $\AA$; the bond angles are kept at $120.2^{\circ}$.

which led to $t_{d}=-4.87 \mathrm{eV}$ and $t_{s}=-2.38 \mathrm{eV}$ for PMI. Another approximation was proposed by Schulten and co-workers, ${ }^{8}$

$$
t_{i j}=-2.6+3.21\left(r_{i j}-1.397\right) \mathrm{eV},
$$

which led to $t_{d}=-3.03 \mathrm{eV}$ and $t_{s}=-2.62 \mathrm{eV}$ for PMI. Using the above two approximations, Jacquemin and co-workers ${ }^{29}$ obtained the values for the static first hyperpolarizability $\beta$ that differ in magnitude and in sign. The CDMV method developed in this paper provides a systematic approach to determine the effective Hamiltonian. It allows us to incorporate the effective electron-electron interaction in a self-consistent manner. Whether the CDMV method implies a certain kind of semi-empirical relation between $t_{i j}$ and $r_{m n}$ is yet to be answered. However, we have found there may be a linear relation between the difference of the single and double bond resonance integrals and the bond length alternation (BLA). To investigate the structure effects on the effective Hamiltonian, we vary the geometry of PMI $(-\mathrm{CH}=\mathrm{N}-)_{10}$ oligomer by changing the double and single bond lengths. The bond angles are kept the same. Table VII lists five of the PMI structural variations, denoted as $\mathbf{a}, \mathbf{b}, \mathbf{c}, \mathbf{d}$ and $\mathbf{e}$, which have the same BLA $\delta R \equiv R_{\mathrm{C}-\mathrm{N}}$ $-R_{\mathrm{C}=\mathrm{N}}$ as those studied in Ref. 28. The effective Hamiltonians and the optical gaps $\Delta$ are determined for these structures, and the results are tabulated in Table VIII. Figure 2 plots $\delta t \equiv t_{s}-t_{d}$ versus $\delta R$ in dots together with the best linear fit. We obtain for a PMI $(-\mathrm{CH}=\mathrm{N}-)_{10}$ oligomer,

$$
\delta t / \mathrm{eV}=0.30+4.90 \times(\delta R / \AA) .
$$

As can be seen from Tables VII and VIII, the optical gap $\Delta$ also increases as the BLA $\delta R$ increases. The possibility of using the present CDMV effective Hamiltonian approach to incorporate the vibrational dynamics of conjugated polymer via its structural variations will be investigated in the future.

To summarize, we have proposed and implemented a new variation method (CDMV) to determine the effective Hamiltonians for $\pi$ electrons in conjugated polymers. This

TABLE VIII. Effective Hamiltonians for five structural variations of the PMI oligomer $(-\mathrm{CH}=\mathrm{N}-)_{10} \cdot t_{d}$ and $t_{s}$ are the average hopping matrix elements for $\mathrm{C}=\mathrm{N}$ and $\mathrm{C}-\mathrm{N}$, respectively. $t^{*}(\mathrm{C})$ and $t^{*}(\mathrm{~N})$ are the average effective atomic orbital energies for $\pi$ orbitals of $\mathrm{C}$ and $\mathrm{N}$ atoms, respectively. $\delta t \equiv t_{d}-t_{s} . \Delta$ is the optical gap.

\begin{tabular}{cccccc}
\hline \hline & $t_{d} / \mathrm{eV}$ & $t_{s} / \mathrm{eV}$ & $\delta t / \mathrm{eV}$ & $t^{*}(\mathrm{C})-t^{*}(\mathrm{~N}) / \mathrm{eV}$ & $\Delta / \mathrm{eV}$ \\
\hline $\mathbf{a}$ & -3.04 & -2.25 & -0.79 & 1.19 & 3.07 \\
$\mathbf{b}$ & -3.11 & -2.19 & -0.92 & 1.18 & 3.35 \\
$\mathbf{c}$ & -3.16 & -2.12 & -1.04 & 1.17 & 3.61 \\
$\mathbf{d}$ & -3.25 & -2.01 & -1.23 & 1.16 & 4.01 \\
$\mathbf{e}$ & -3.33 & -1.95 & -1.38 & 1.15 & 4.33 \\
\hline \hline
\end{tabular}

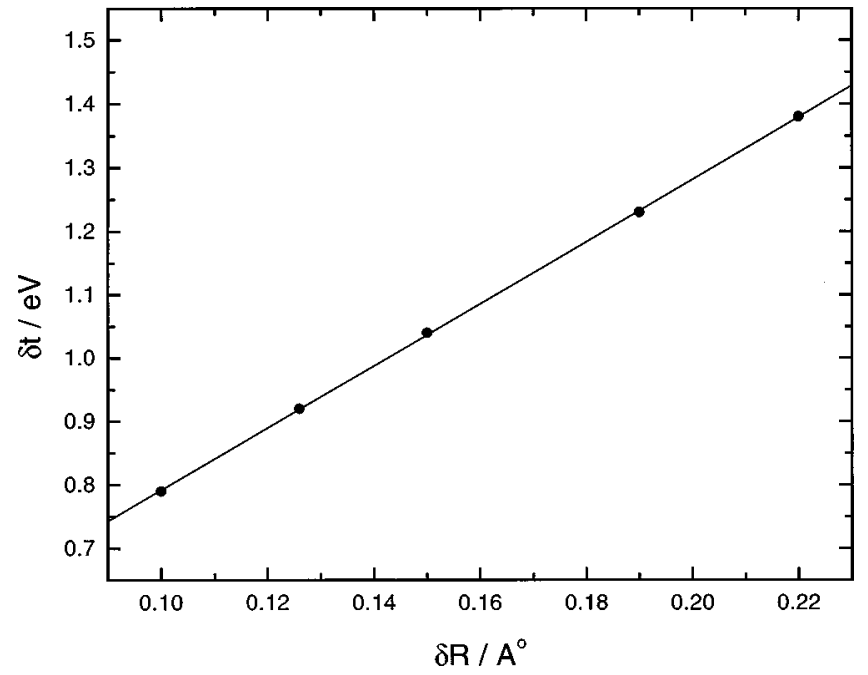

FIG. 2. The relationship between $\delta t \equiv t_{s}-t_{d}$ and $\delta R=R_{s}-R_{d}$ for the PMI $(-\mathrm{CH}=\mathrm{N}-)_{10}$ oligomer. The line is the best linear fit to the data (dots).

CDMV approach has been applied successfully to several PMI, PAE and PAZ oligomers. Compared to the approach employed in Ref. 19, the CDMV approach is more general, and applicable to a wider spectrum of molecules.

\section{ACKNOWLEDGMENTS}

We thank W. Liang for her assistance in preparing the figures. The support of the Committee on Research and Conference Grants of the University of Hong Kong and the Earmarked Grant from the Research Grant Council of Hong Kong Government is gratefully acknowledged.

${ }^{1}$ J. C. Phillips and L. Kleinman, Phys. Rev. 116, 287 (1959).

${ }^{2}$ J. D. Weeks, A. Hazi, and S. A. Rice, Adv. Chem. Phys. 16, 283 (1969).

${ }^{3}$ C. F. Melius, I. W. A. Goddard, and L. R. Kahn, J. Chem. Phys. 56, 3342 (1971).

${ }^{4}$ L. R. Kahn, P. Baybutt, and D. G. Truhlar, J. Chem. Phys. 65, 3826 (1976).

${ }^{5}$ R. Pariser and R. G. Parr, J. Chem. Phys. 21, 767 (1053).

${ }^{6}$ J. A. Pople, Trans. Faraday Soc. 49, 1375 (1953).

${ }^{7}$ H. Fukutome, J. Mol. Struct.: THEOCHEM 188, 337 (1989).

${ }^{8}$ K. Schulten, I. Ohmine, and M. Karplus, J. Chem. Phys. 64, 4422 (1976).

${ }^{9}$ G. Segal and J. A. Pople, J. Chem. Phys. 46, 3286 (1966).

${ }^{10}$ J. A. Pople, D. Beveridge, and P. Dobosh, J. Chem. Phys. 47, 2026 (1967).

${ }^{11}$ M. Dewar and W. Thiel, J. Am. Chem. Soc. 99, 4499 (1977).

${ }^{12}$ M. J. S. Dewar, E. G. Zeebisch, E. F. Healy, and J. J. P. Stewart, J. Am. Chem. Soc. 107, 3902 (1985).

${ }^{13}$ B. H. Brandow, Int. J. Quantum Chem. 35, 207 (1979).

${ }^{14}$ C. H. Martin and K. F. Freed, J. Chem. Phys. 101, 4011 (1994).

${ }^{15}$ S. Priyadarshy, S. S. Skourtis, S. M. Risser, and D. N. Beratan, J. Chem. Phys. 104, 9473 (1996).

${ }^{16}$ I. V. Kurnikov and D. N. Beratan, J. Chem. Phys. 105, 9561 (1996).

${ }^{17}$ G. H. Chen and S. Mukamel, J. Chem. Phys. 103, 9355 (1995).

${ }^{18}$ P. Hohenberg and W. Kohn, Phys. Rev. B 136, B864 (1964).

${ }^{19}$ G. H. Chen and S. Mukamel, Chem. Phys. Lett. 258, 589 (1996).

${ }^{20}$ R. M. Gavin and S. A. Rice, J. Chem. Phys. 60, 3231 (1974).

${ }^{21}$ J. A. Pople, Approximate Molecular Orbital Theory (McGraw-Hill, New York, 1970).

${ }^{22}$ G. H. Chen and S. Mukamel, J. Am. Chem. Soc. 117, 4945 (1995).

${ }^{23}$ K. Ohno, Theor. Chim. Acta 2, 219 (1964).

${ }^{24}$ A. E. Reed, L. A. Curtis, and F. Weinhold, Chem. Rev. 88, 899 (1988).

${ }^{25}$ P.-O, Lowdin, Adv. Quantum Chem. 5, 185 (1970).

${ }^{26}$ F. Sondheimer, D. A. Ben-Efraim, and R. Wolovsky, J. Am. Chem. Soc. 83, 1675 (1961). 
${ }^{27}$ A. Takahashi and S. Mukamel, J. Chem. Phys. 100, 2366 (1994).

${ }^{28}$ B. Champagne, D. Jacquemin, J.-M. Andre, and B. Kirtman, J. Phys. Chem. A 101, 3158 (1997).

${ }^{29}$ D. Jacquemin, B. Champagne, J.-M. Andre, and B. Kirtman, Chem. Phys. 213, 217 (1996).
${ }^{30}$ A. K. Bakhshi and J. Ladik, Solid State Commun. 60, 361 (1986).

${ }^{31}$ J. Chandrasekhar and P. K. Das, J. Phys. Chem. 96, 679 (1992).

${ }^{32}$ J. L. Bredas, B. Themans, and J. M. Andre, J. Chem. Phys. 78, 6137 (1983).

${ }^{33}$ G. H. Chen, Z. M. Su, Z. W. Shen, and Y. J. Yan (to be submitted). 